\title{
ROLE OF IMAGING IN MANAGEMENT OF PULMONARY TUBERCULOSIS.
}

Chandrajeet Yadav, Aman Gupta, Amit Tiwari, Prashant Musale.

1. Assistant Professor, Department of Radiodiagnosis. SAIMS Medical College and P.G. Institute, Indore.

2. Professor \& Head, Department of Radiodiagnosis. SAIMS Medical College and P.G. Institute, Indore.

3. Resident, Department of Radiodiagnosis. SAIMS Medical College and P.G. Institute, Indore.

4. Resident, Department of Radiodiagnosis. SAIMS Medical College and P.G. Institute, Indore.

\section{CORRESPONDING AUTHOR:}

Dr. Aman Gupta.

201, Dilpasand Avenue,

5/2, Manoramaganj,

Indore-(M.P.)-452001.

E-mail: aman_sono@yahoo.co.in

ABSTRACT: Pulmonary tuberculosis is probably as old as mankind itself, still it remains the major health problem globally and specially in developing nations. It is estimated that about $40 \%$ of the Indian population is infected with Tubercle Bacillus bacteria, the vast majority of whom have latent rather than active TB.As pulmonary tuberculosis is infectious in nature, early initiation of treatment to render it noninfectious is necessary. Several laboratory investigation techniques have been devised for early and accurate detection of $\mathrm{TB}$, but either they are time consuming or very expensive. Radiodiagnosis is playing an important role in the management of tuberculosis since the discovery of X-rays in 1895. With advent of newer imaging modalities such as USG, CT and MRI and their widespread availability the contribution of radiodiagnosis in management of tuberculosis has been significantly increased.

Chest radiographs play a major role in the screening, diagnosis, and follow-up of the patients with TB. However, the radiographs may be normal or show only mild or nonspecific findings in patients with active disease. CT is more sensitive than chest radiography in the detection and characterization of both subtle localized or disseminated parenchymal disease and mediastinal lymphadenopathy. Familiarity with the patterns of imaging characteristics of pulmonary tuberculosis is needed for its early detection and further management.

INTRODUCTION: The Pulmonary tuberculosis is probably as old as mankind itself. But it took several centuries to discover Mycobacterium tuberculosis as a causative agent by Robert Koch in 1882. The discovery of streptomycin in 1944, para-amino salicylic acid in 1946 and isoniazid in1952 led to the first effective cure for TB. And the descriptions of airborne transmission of infection and of reactivation of dormant infection in the 1960s by Riley and Stead and Colleagues respectively, furthered our understanding of the spread and pathogenesis of this disease. ${ }^{1}$ But TB still remains the major health problem globally and specially in developing nations.

It is estimated that about $40 \%$ of the Indian population is infected with TB bacteria, the vast majority of whom have latent rather than active TB. India is the highest TB burden country with World Health Organization (WHO) statistics for 2011 giving an estimated incidence figure of 2.2 million cases of TB for India out of a global incidence of 8.7 million cases. The estimated TB prevalence figure for 2011 is given as 3.1 million. ${ }^{2}$ 
If TB is detected early and fully treated, people with the disease quickly become noninfectious and eventually cured. However, multidrug-resistant (MDR) and extensively drugresistant TB, HIV-associated TB, and weak health systems are major challenges. The World Health Organization is making an effort to dramatically reduce the burden of TB and TB deaths and prevalence by 2015, through its Stop TB Strategy and supporting the Global Plan to Stop TB. ${ }^{3}$

Several laboratory investigation techniques have been devised for early and accurate detection of TB, but either they are time consuming or very expensive. Recently "The Xpert MTB/RIF test" has been claimed a rapid molecular test that can diagnose TB and rifampicin resistance within 100 minutes. ${ }^{4}$ But according to 'Deputy Director General, Central TB control Division, Ministry of Health and Family Welfare', the technique cannot be used for routine testing in India, as here the climate and temperature conditions are different from the western countries. Solid culture/sputum testing remains the golden standard for Indian conditions. ${ }^{4}$ Medical laboratory clinicians are working for an ideal diagnostic method and we hope one in future.

Radiodiagnosis plays an important role in the management of tuberculosis since the discovery of X-rays in 1895. With advent of newer imaging modalities such as USG, CT and MRI and their widespread availability, the contribution of radio-diagnosis in management of tuberculosis has been significantly increased. Familiarity with the patterns of imaging characteristics of pulmonary tuberculosis is of great help in its early detection. As pulmonary tuberculosis is infectious in nature, early initiation of treatment to render it noninfectious is necessary. The present study was undertaken to evaluate the patterns of imaging characteristics in known cases of pulmonary tuberculosis.

MATERIALS AND METHODS:A prospective study was carried out in Department of Radiodiagnosis, SAIMS Medical College\& PG Institute, Indore from January 2011 to February 2012.Informed,written consent was taken from all the patients. Patients were selected from either the Outpatients Department (OPD), or from those admitted to the Chest and TB Ward. Patients with known malignancy, cardiac disease and co-infection with other organisms were excluded from the study. A total of 100 patients were selected from a larger pool of chronic pulmonary disease after being clinically diagnosed as pulmonary tuberculosis. These patients were subjected to detailed history taking through a well-designed and comprehensive proforma which included recording age, sex, weight, socioeconomic status, smoking habits and HIV status. They were later clinically evaluated for a battery of signs and symptoms. Details of laboratory tests including Hemoglobin, ESR, TLC, DLC and sputum for AFB and HIV were recorded. The patients with established diagnosis of pulmonary tuberculosis were asked to bring their previous imaging studies and if required they were subjected to chest radiography and/or CT scanning.

Frontal radiograph (postero-anterior view) of chest was taken in deep inspiration with proper patient positioning and using high $\mathrm{kVp}$ technique on 300mA G.E. SRS300 machine.

Chest CT examinations were performed on Siemens Somatom64-slice multidetectorscanner. The images were obtained at end-inspiration and viewed at window settings optimized for assessment of lung parenchyma (window width 1500-1800 HU; window level 500$700 \mathrm{HU}$ ) and mediastinal structures (window width $400 \mathrm{HU}$; window level $40 \mathrm{HU}$ ). Intravenous contrast medium was administered only when considered useful.

Every Chest radiograph and CT chest were interpreted by one of the co-author radiologists. 
Radiographical and CT imaging findings were recorded. After recording imaging findings, lobar location and the side of involvement were notified. Statistical analysis was performed by SPSS 8 computer program.

OBSERVATIONS AND RESULTS: The study population comprised of 100 patients. Majority of patients in our study were in age group 11-30yrs (46\%).There was no significant sex predilection. Most of the patients were belonging to low income group (Table-1). Approximately $78 \%$ of patients had history of weight loss (Table-2). The most common presenting symptom was productive cough (98\%) with fever, night sweats, anorexia and chest pain as other common symptoms (Table-3). Patients tend to present within 1 to 3 months of the start of their disease related symptoms, with a majority (89\%) presenting by six months (Table-4).

Laboratory data showed anemia of mild to moderate degree in all cases, with a mild leukocytosis. The ESR was raised in $95.2 \%$ of cases (Table-5).Sputum smears for AFB was positive in approx. $51 \%$ of the cases (Table-6).

The most common abnormality on a chest radiograph in both genders was pulmonary consolidation-infiltration,(Figure-1) which was seen in $56 \%$ of the cases. It has been reported as the most common finding in other studies as well.5Single lobe involvement was seen in $66 \%$ of cases. If only one segment was involved, the distribution of infiltration was as followsRight upper lobe (RUL) in 32\%, left upper lobe (LUL) in $28 \%$, right lower lobe (RLL) in $12 \%$, right middle lobe (RML) in 9\%, left lower lobe (LLL) in $8 \%$ and lingual and mid zone of the left lung were involved in $11 \%$ of the cases. There was clear upper lobe predilection of disease with upper lobe involvement in 60\%of patients. Broncho-pneumonic and both lung involvement was seen in $23 \%$ and $11 \%$ of the cases, respectively. Other radiographical findings were as follows: fibrosis in 23\%,(Figure-5) pleural effusion in 38\%,(Figure-4) cavitatory lesionin $22 \%$ of the cases;(Figure-2,3a) which appeared as thick-wall cavity with air-fluid level in 2\%.Pleural thickening was seen in 19\% cases; of which 2 cases of pleural thickening(10.5\%) contained calcifications(2\% of all cases).Bronchiectasis was detected in $22 \%$, lymphadenopathy in $8 \%$; of which three(37.5\%) revealed calcifications (3\% of all cases). Miliary pattern was noticed in $3 \%$ of all cases.(Table-7)

Right lung involvement was seen in 53\% and left lung involvement was noticed in $47 \%$ of the cases; however, these differences were not significant $(p=0.056)$.Pleural effusion was slightly more common on the right side. Some rare radiographical findings were calcified granuloma in $9 \%$, emphysematous changes in 7\%, pneumothorax in $4 \%$ ( Figure-6) and collapse in $2 \%$. (Table-7) Among the different patients, HRCT findings of active PTB, centrilobular nodules were noted in 91\% cases,(Figure-8a) lobular consolidation in 38\%, (Figure-8b) cavitation in 48\%(Figure-7)and 'tree-in-bud' appearance in 88\%(Figure-7a,8a).

Lymphadenopathy (Fig-10a) and miliary nodules(Figure-10b) were seen in 16\% and 3\% cases respectively. Four patients with lymphadenopathy showed non-enhancing lymph nodes, while central hypo-attenuation was seen in six cases. None had complete normal HRCT chest (Table-8).Unilateral lesions in right and left lung fields were noted in 53\% and $42 \%$ cases respectively on X-ray chest as compared to $12 \%$ and $7 \%$ cases on HRCT respectively. $5 \%$ had involvement of both lung fields on Chest radiograph as compared to $81 \%$ on HRCT. The difference was found to be statistically significant $(p<0.05)$. Cavitation was detected in $48 \%$ cases on HRCT as 
compared to $22 \%$ patients on X-ray chest $(\mathrm{p}<0.05)$.

Table-1: Basic Demographic data of patients $(n=100)$

\begin{tabular}{|l|l|l|}
\hline Demographic Data & Variables & $\begin{array}{l}\text { No. of Patients } \\
(\mathrm{n}=100)\end{array}$ \\
\hline \multirow{3}{*}{$\begin{array}{l}\text { Age Wise distribution of patients. } \\
\text { (in years) }\end{array}$} & $11-30 \mathrm{yrs}$ & 46 \\
\cline { 2 - 3 } & $31-50 \mathrm{yrs}$ & 18 \\
\cline { 2 - 3 } & $51-70 \mathrm{yrs}$ & 28 \\
\cline { 2 - 3 } & $>70 \mathrm{yrs}$ & 08 \\
\hline \multirow{3}{*}{ Sex wise distribution of cases } & Male & 48 \\
\cline { 2 - 3 } & Female & 52 \\
\hline \multirow{2}{*}{$\begin{array}{l}\text { Distribution on basis of monthly income(Rs. } \\
\text { per month) }\end{array}$} & $<4000$ & 48 \\
\cline { 2 - 3 } & $4000-8000$ & 32 \\
\cline { 2 - 3 } & $8000-12000$ & 14 \\
\cline { 2 - 3 } & $>12000$ & 06 \\
\hline
\end{tabular}

Table-2: Distribution of patients on basis of weight loss $(n=100)$

\begin{tabular}{|l|l|}
\hline History of Weight Loss & $\%$ (percentage) \\
\hline Weight Loss: & 78 \\
\hline No weight loss: & 22 \\
\hline
\end{tabular}

Table-3: Classification of patients on basis of symptoms and their

frequency:- $(n=100)$

\begin{tabular}{|l|l|}
\hline Symptoms & Frequency \\
\hline Cough & $98 \%$ \\
\hline Fever & $96 \%$ \\
\hline Sputum production & $85 \%$ \\
\hline Night Sweats & $63 \%$ \\
\hline Anorexia & $61 \%$ \\
\hline Chest Pain & $59 \%$ \\
\hline Clubbing & $08 \%$ \\
\hline Hemoptysis & $11 \%$ \\
\hline Peripheral lymphadenopathy & $04 \%$ \\
\hline
\end{tabular}


Table-4: Distribution on basis of duration of disease at the time of presentation:- $(n=100)$

\begin{tabular}{|l|l|}
\hline Duration (in days) & Number of patients. \\
\hline$<30$ & 21 \\
\hline $31-90$ & 44 \\
\hline $91-180$ & 24 \\
\hline $181-365$ & 08 \\
\hline$>365$ & 03 \\
\hline
\end{tabular}

Table-5: Distribution of patients on basis of hematological data.

\begin{tabular}{|c|c|c|}
\hline \multirow[t]{2}{*}{ Test } & \multicolumn{2}{|c|}{ Values } \\
\hline & Results & Numbers \\
\hline $\begin{array}{l}\mathrm{Hb}(\mathrm{gms} / \mathrm{dl}) \\
(\mathrm{n}=100)\end{array}$ & $\begin{array}{l}7-9 \\
9.1-11 \\
11.1-13 \\
>13\end{array}$ & $\begin{array}{l}14 \\
28 \\
51 \\
07\end{array}$ \\
\hline $\begin{array}{l}\text { ESR } \\
(\mathrm{mm} / 1 \mathrm{st} \text { hour }) \\
(\mathrm{n}=100)\end{array}$ & $\begin{array}{l}<5 \\
6-30 \\
31-55 \\
56-80 \\
81-105 \\
>106\end{array}$ & $\begin{array}{l}04 \\
18 \\
30 \\
32 \\
14 \\
02\end{array}$ \\
\hline $\begin{array}{l}\text { TLC } \\
\text { (count / cu mm) } \\
(n=100)\end{array}$ & $\begin{array}{l}3-5 \\
5.1-7 \\
7.1-9 \\
9.1-11 \\
>11\end{array}$ & $\begin{array}{l}28 \\
36 \\
18 \\
09 \\
09\end{array}$ \\
\hline $\begin{array}{l}\text { DLC } \\
\text { Polymorphs }(\%) \\
(n=100)\end{array}$ & $\begin{array}{l}50-60 \\
61-70 \\
71-80 \\
>80\end{array}$ & $\begin{array}{l}36 \\
31 \\
29 \\
04\end{array}$ \\
\hline $\begin{array}{l}\text { Lymphocytes }(\%) \\
(\mathrm{n}=100)\end{array}$ & $\begin{array}{l}\text { Up to } 10 \\
11-20 \\
21-30 \\
31-40 \\
41-50\end{array}$ & $\begin{array}{l}04 \\
14 \\
41 \\
27 \\
14\end{array}$ \\
\hline
\end{tabular}


Table-6: Distribution of patients on basis of Sputum AFB results.

\begin{tabular}{|c|c|c|c|}
\hline \multirow[t]{2}{*}{ Specimen for AFB stain } & \multicolumn{3}{|c|}{ Result } \\
\hline & Positive & & Negative \\
\hline \multirow[t]{2}{*}{ Sputum $(n=96)$} & & $\begin{array}{l}49 \\
(51.05 \%)\end{array}$ & $\begin{array}{c}47 \\
(48.95 \%)\end{array}$ \\
\hline & $\begin{array}{l}\text { Scanty } 10 \\
\text { One plus } 13 \\
\text { Two plus11 } \\
\text { Three plus15 }\end{array}$ & $\begin{array}{l}(20.40 \%) \\
(26.53 \%) \\
(22.44 \%) \\
(30.63 \%)\end{array}$ & \\
\hline $\begin{array}{l}\text { No Sputum } \\
(\mathrm{n}=4)\end{array}$ & \multicolumn{2}{|l|}{-} & - \\
\hline
\end{tabular}

Table-7: Classification of patients on basis of Chest X-ray findings.

\begin{tabular}{|l|l|}
\hline Patten Of Lesion & No. of patients \\
\hline Normal & 9 \\
\hline Infiltrates & 56 \\
\hline Fibrosis & 23 \\
\hline Consolidation & 19 \\
\hline Cavitation & 22 \\
\hline Miliary pattern & 03 \\
\hline Hilar LAD & 02 \\
\hline Bronchiectasis & 22 \\
\hline Collapse & 02 \\
\hline Pneumothorax & 04 \\
\hline Effusion & 38 \\
\hline Calcified granuloma & 09 \\
\hline Pleural thickening & 19 \\
\hline Emphysematous changes & 07 \\
\hline
\end{tabular}


Table-8: Distribution of patients on basis of Computed tomography findings.

\begin{tabular}{|l|l|}
\hline Pattern of Lesion & No.of Patients \\
\hline Centrilobular nodules & 91 \\
\hline Tree- in- bud opacities & 92 \\
\hline Bronchial wall thickening & 71 \\
\hline Cavities & 48 \\
\hline Bronchiectasis & 34 \\
\hline Miliary nodules & 03 \\
\hline Fibrosis & 38 \\
\hline Collapse & 02 \\
\hline Mediastinal and hilar lymphadenopathy & 16 \\
\hline Pleural effusion & 42 \\
\hline Pleural thickening & 23 \\
\hline Pneumothorax & 04 \\
\hline Pneumomediastinum & 00 \\
\hline Calcified granuloma & 12 \\
\hline Emphysematous changes & 11 \\
\hline Normal & 00 \\
\hline
\end{tabular}

DISCUSSION: Tuberculosis is a chronic granulomatous infection characterized by caseation necrosis and great propensity for fibrosis and calcification. It is caused by Mycobacterium tuberculosis- a strictly aerobic, acid fast rod shaped bacillus. M tuberculosis transmitted via airborne droplet nuclei that are produced when persons with pulmonary or laryngeal TB cough, sneeze, speak, or sing. The particles, which measure 1-5 $\mu \mathrm{m}$ in size, can be kept airborne by normal air currents for prolonged periods of time, resulting in dispersion throughout a room or building. The presence of acid-fast bacilli in the sputum smear is the main indicator of potential for transmission other source patient characteristics that increase the probability of transmission include positive sputum culture for M. tuberculosis, presence of cavitations on the chest radiograph, presence of TB laryngitis, and high-volume and watery respiratorysecretions. ${ }^{6}$

The patients of pulmonary tuberculosis show distinct subjective and objective features that allow their differentiation from other acute or chronic pulmonary diseases. In this study, the common features at presentation were productive cough with fever, weight loss, night sweats, anorexia and chest pain. Clubbing was observed in $8 \%$ of our cases with a known predilection for bronchiectasis and chronic fibrosis. These findings were present in a bimodal distribution with a younger age group of less than 30 years and an older group of between 51-70 years. Our presenting symptoms are in concordance with those established by the WHO.

A majority of patients (approximately 48\%) belonged to the poor socio-economic group with a mean monthly income of less than Rs. 4000. As they are predisposed to malnutrition of several types, and perhaps has a poor immunity. It was observed that patients often tend to present late, 
with a majority within 1 to 3 months or by six months of the start of their disease-related symptoms. As pulmonary symptoms tend to be attributed to other conditions like colds and flu, that patients wait for the disease to become more advanced before seeking medical attention.

Laboratory data showed anemia of mild to moderate degree in all cases, with a mild leukocytosis, others have showed this as well. ${ }^{7}$ The ESR was raised in $95.2 \%$ of cases.

Chest radiographs play a major role in the screening, diagnosis, and follow-up of the patients with TB. However, the radiographs may be normal or show only mild or nonspecific findings in patients with activedisease. ${ }^{8}$ Common causes of a missed diagnosis of TB are failure to recognize hilar and mediastinal lymphadenopathy as a manifestation of primary disease in adults, overlooking of mild parenchymal abnormalities in patients with reactivation disease, and failure to recognize that an upper lobe nodule or mass surrounded by small nodular opacities or scarring may represent TB. ${ }^{8}$ CT is more sensitive than chest radiography in the detection and characterization of both subtle localized or disseminated parenchymal disease and mediastinal lymphadenopathy. 9 The radiographic diagnosis of TB is initially correct in only $49 \%$ of all cases;34\% for the diagnosis of primary TB and59\% for the diagnosis of reactivation TB. 5 With CT, the diagnosis of pulmonary TB is correct in $91 \%$ of patients and TB is correctly excluded in $76 \%$ of patients. ${ }^{10}$

High resolution CT is particularly helpful in the detection of small foci of cavitation in areas of confluent pneumonia and in areas of dense nodularity and scarring. ${ }^{8}$ Tuberculous cavity develops within a consolidated segment as the caseation necrosis erodes into the bronchial tree, expelling liquefied debris. ${ }^{11}$ On HRCT, cavities due to TB can be thick or thin walled and may have smooth or irregular margins. ${ }^{12}$ Thick walled cavities probably represent early stages of necrotizing consolidation and may resolve completely, leave a scar, become thin walled or remain open at the end of treatment. ${ }^{12}$ However, disease activity cannot be determined on the basis of CT appearance of the cavity alone, and diagnosis must be made from the results of sputum cultures and serial radiographic analysis. ${ }^{12}$ Presence of multiple cavities (especially if more than three) in an area of consolidation is found more frequently in PTB than in any other lesion. ${ }^{13}$ HRCT is more sensitive than plain radiography in the detection of small cavities, particularly at the apices, lung bases, paramediastinal and retrocardiac locations. ${ }^{12}$

HRCT is more sensitive than chest radiograph in differentiating between active and inactive tuberculous lesions. And also in detecting endobronchial spread, it is far better having 98\% sensitivity as compared to $19-58 \%$ on chest radiograph. ${ }^{10,14}$ HRCT findings in active tuberculosis include patchy, frequently peribronchial, unilateral or bilateral airspace consolidation, cavitationthick or thin walled, scattered airspace nodules, centrilobular branching structures / 'tree-in-bud' appearance, miliary disease, pleural effusion, empyema and bronchopleural fistula, and low density hilar/mediastinal lymphadenopathy. ${ }^{11}$ Lesions in and around the small airways are the most characteristic CT feature of early active tuberculosis and a heterogeneous, poorly marginated opacity/ consolidation, usually in apical or posterior segment of upper lobes and superior/apical segments of lower lobes is the earliest finding of postprimary PTB. ${ }^{9}$

In our patients centrilobular nodules were the most common finding (91\%), which was similar to the reported prevalence of $91 \%, 92 \%$ and $92 \%$ by Lee et al., Raniga et al. and Nhatipoglu et al.respectively. ${ }^{9,10,14}$ Lobular consolidations were noted in $38 \%$ of cases, which is comparable to the studies by Lee et al. (52\%), Raniga et al. (52\%) and Nhatipoglu et al (44\%) . 9,10,14Cavitation was 


\section{ORIGINAL ARTICLE}

observed in $48 \%$ of patients in our study as compared to $51 \%, 64 \%$ and $50 \%$ cases respectively in the above studies. 'Tree-in-bud' appearance was noted in $88 \%$ as compared to $71 \%$ cases in study by Nhatipogluet al. 15 The incidence of mediastinal lymphadenopathy (16\%) and miliary nodules (3\%) was similar to the abovementioned studies . 9,10,14

Pulmonary tuberculosis has been traditionally divided into primary and post-primary tuberculosis, with primary tuberculosis being considered a disease of childhood and post-primary tuberculosis is a disease of adulthood. Primary TB is acquired by the inhalation of airborne organisms. In primary TB, because patients are not sensitized to tubercle bacilli, immune reaction for localization of the lesion and killing of the bacilli is lacking. Inability to localize the lesion results in a wide area of airspace consolidation accompanied by infiltration of neutrophils and occurrence of caseation necrosis. Until the cellular immune response develops, infection can progress locally and spread beyond the area of primary focus. Lymphatic spread of organisms to hilar and mediastinal lymph nodes occur, along with a frequent, usually subclinical, hematogenous spread. Healing of larger parenchymal lesions may leave fibrous scars or persistent nodules known as tuberculomas, both of which may calcify. ${ }^{15}$

Post primary TB results from reactivation of a previously dormant primary infection in $90 \%$ of cases; a minority of cases represents a continuation of the primary disease. Exogenous reinfection rarely occurs. Despite the development of specific immunity resulting in healing with fibrosis or the granulomas, viable organisms often survive. Reactivation of dormant bacilli occurs during periods of immune depression, malnutrition, and debilitation, or as a result of aging. Pulmonary lesions tend to be localized forming predominantly nodular lesions rather than wide areas of consolidation. The capability to localize the tuberculous lesion is provided by sensitization of the host by previous infection. Unlike primary TB, which is often an acute and self- limited disease, postprimary TB is typically a chronic, slowly progressive disease with high morbidity and mortality if not adequately treated. Although the radiographic findings of postprimary TB may overlap those of primary TB, distinguishing features include a predilection for the upper lobes, absence of CT lymphadenopathy, and a propensity for cavitation. ${ }^{15}$

The prevalence of primary tuberculosis in adults is increasing, however because primary tuberculosis is perceived to be a disease of childhood, it is often not suspected in adults, resulting in misdiagnosis. ${ }^{16}$ It can sometimes be difficult to differentiate between primary and post-primary tuberculosis both clinically and radiologically, since their features can overlap. However, confirming the diagnosis is more important than identifying the subtype because it allows initiation of correct clinical management.

CONCLUSION: The correct diagnosis and determination of disease activity are two important issues to be addressed for pulmonary tuberculosis. There are no specific clinical diagnostic criteria to diagnose pulmonary tuberculosis with confidence. It usually depends on the detection of acid-fast bacilli on culture of sputum or bronchoalveolar lavage fluid. Acid-fast bacilli, however, are found in a limited number of patients (20\% to 55\%) with active pulmonary TB, in particular in patients with AIDS. And hence the radio-imaging has to play a key role in the timely diagnosis and management of pulmonary tuberculosis patients.

Chest radiographs are important not only in diagnosis but also in follow-up of treatment response. However, disease activity cannot be assessed accurately by chest radiography. Although active 
infection is most often associated with exudative lesions or cavitation on chest radiographs, fibroproductive lesions also indicate active disease. CT scans, especially HRCT scans, are superior to chest radiographs for assessing the disease activity of postprimary TB. In appropriate clinical settings with atypical or seemingly inactive X-ray chest features and smear negative PTB; the clinician can reach the presumptive diagnosis of active tuberculosis and start empirical therapy. Familiarity with the patterns of imaging characteristics of pulmonary tuberculosis is needed for its early detection and further management.

\section{REFERENCES:}

1. Leung AN. Pulmonary tuberculosis: the essentials. Radiology 1999; 198:307-22. http://www.who.int/tb/country/data/profiles/en/. WHO Website. Accessed March 25, 2013.

2. World Health Organization. Programmes and projects. Tuberculosis. The Stop TB Strategy. www.who.int/tb/strategy/en/. WHO Website.Accessed March 25, 2013.

3. World Health Organization. Programmes and projects. Tuberculosis.TB Publications. Tuberculosis global facts.http://www.who.int/tb/publications/actsheets/en/.WHO Website. Accessed March 27, 2013.

4. TB Facts.org. TB Tests. Xpert TB Test. http://www.tbfacts.org/xpert-tbtest.html.Informations about Tuberculosis website. Accessed March 27, 2013.

5. 5. Woodring JH, Vandiviere HM, Fried AM, Dillon ML, Williams TD, Melvin IG. Update: the radiographic features of pulmonary tuberculosis. Am J Roentgenol 1986.Mar;146(3):497-506.

6. American Thoracic Society. Control of tuberculosis in the United States. Am Rev Respir Dis $1992 ; 146: 1623-1632$.

7. Akintunde EO, Shokumbi WA, Adekunle CO. Leucocyte count, platelet count and erythrocyte sedimentation rate in pulmonary tuberculosis. Afr J Med Sci 1995; 24(2):131-4.

8. Im JG, Itoh H, Shim YS, et al. Pulmonary tuberculosis: CT findings-early active disease and sequential change with antituberculoustherapy.Radiology1993; 186: 653-660.

9. Lee KS, Hwang JW, Chung PM, Kim H, Kyun OJ. Utility of CT in the evaluation of pulmonary tuberculosis in patients without AIDS. Chest 1996; 110:977-84.

10. Raniga S, Parikh N, Arora A, Vaghani M, Vora PA, Vaidya V. Is HRCT reliable in determining disease activity in pulmonary tuberculosis? Indian J Radiol Imaging 2006; 16:221-8.

11. Kuhlman JE, Deutsch JH, Fishman EK, Siegelman SS. CT features of thoracic mycobacterial disease. Radiographics 1990; 10:413-31.

12. Kim WJ, Kim SJ, han GS. CT findings of pulmonary consolidation: focused on tuberculosis, malignant obstructive pneumonitis, and lung abscess. J Korean RadiolSoc 1994; 32: 405-10.

13. Christensen EE, Dietz GW, Ahn CH, Chapman JS, Murry RC, Anderson J, et al. Initial roentgenographic manifestations of pulmonary mycobacterium tuberculosis, M. Kansasii and M. intracellularis infections. Chest 1981; 80:132-6.

14. O Nhatipoglu, EOsma, MManisali, ESUqan, PBalci,AAkkoclu, Akpinar, C Karlikaya, C Yuksel High resolution computed tomographic findings in pulmonary tuberculosis, Thorax 1996;51:397-402.

15. Goo JM, Im JG CT of Tuberculous and non-tuberculous Mycobacterial infections. Radiol Clin 


\section{ORIGINAL ARTICLE}

North Am 2002;40-1:655-77.

16. Andreu J, Ca'ceres J, Pallisa E, Martinez-Rodriguez M. Radiological manifestations of pulmonary tuberculosis. Eur J Radiol 2004; 51(2):139-149.

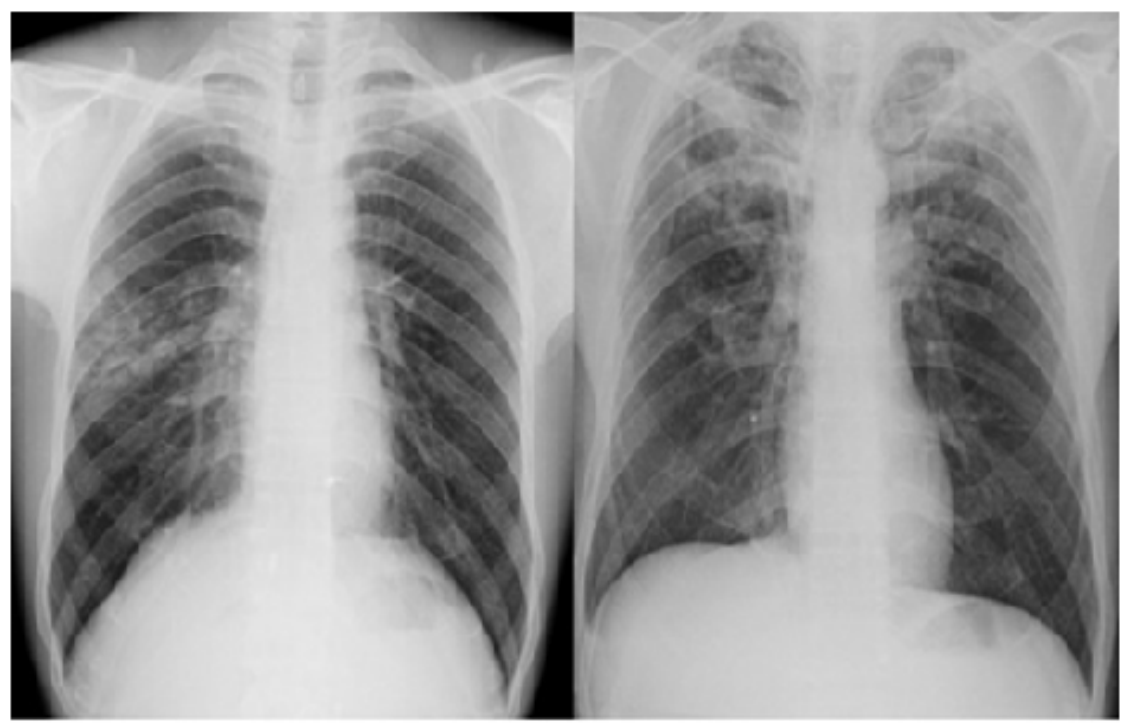

Fig.1(a, b)- X ray Chest PA View in patients sputum positive for Acid fast bacilli showing infiltration - consolidation pattern in right mid zone (a) and bilateral upper zones (b).

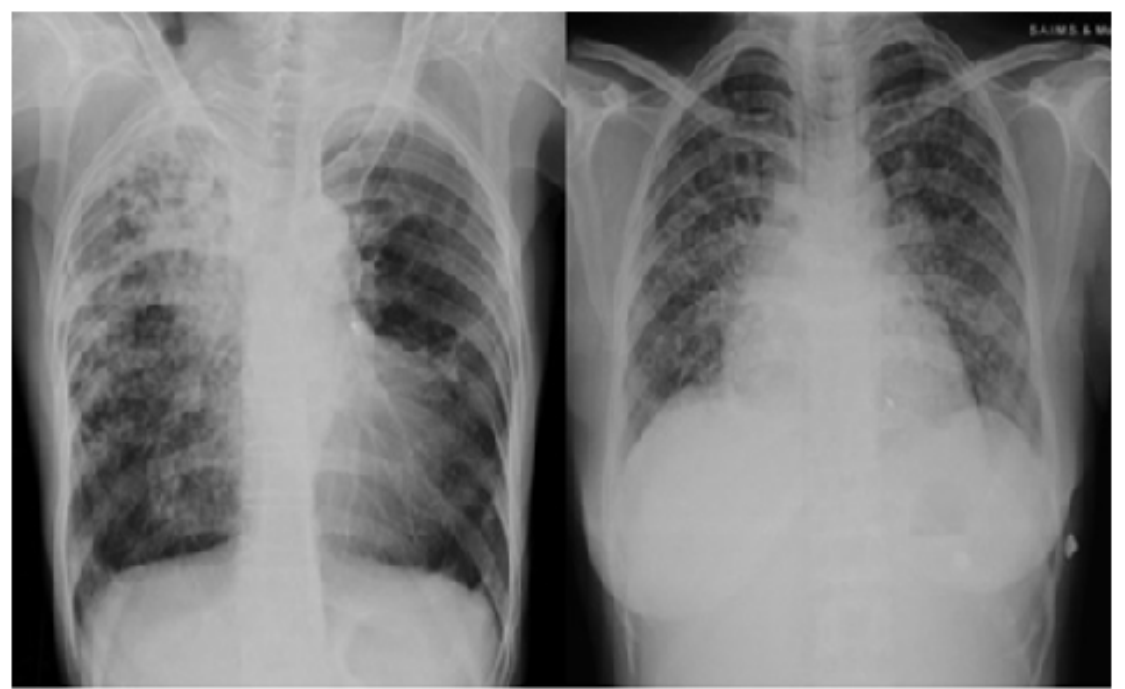

Fig.2 (a, b): Skiagram Chest P.A. View in AFB positive patients.(a) Non-homogenous opacity with cavitation in right upper and both mid zones.

(b)Nodular,opacities bilateral lung fields-Disseminated Koch's. 


\section{ORIGINAL ARTICLE}

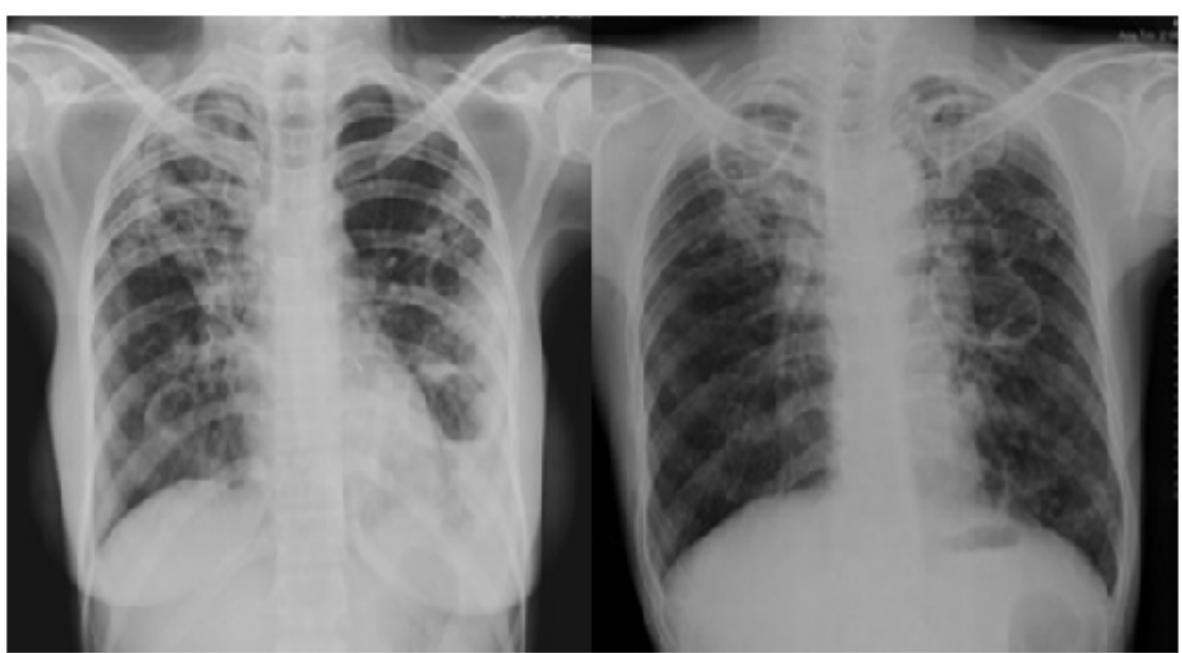

Fig.3 (a, b) X-ray Chest PA view in sputum positive patients (a) Cavitatory lesion right apical region, left mid and bilateral lower zones with surrounding non-homogenous opacities.(b)Cavitatory lesion both upper and left mid zones with scattered calcified spots bilateral upper and mid zones.

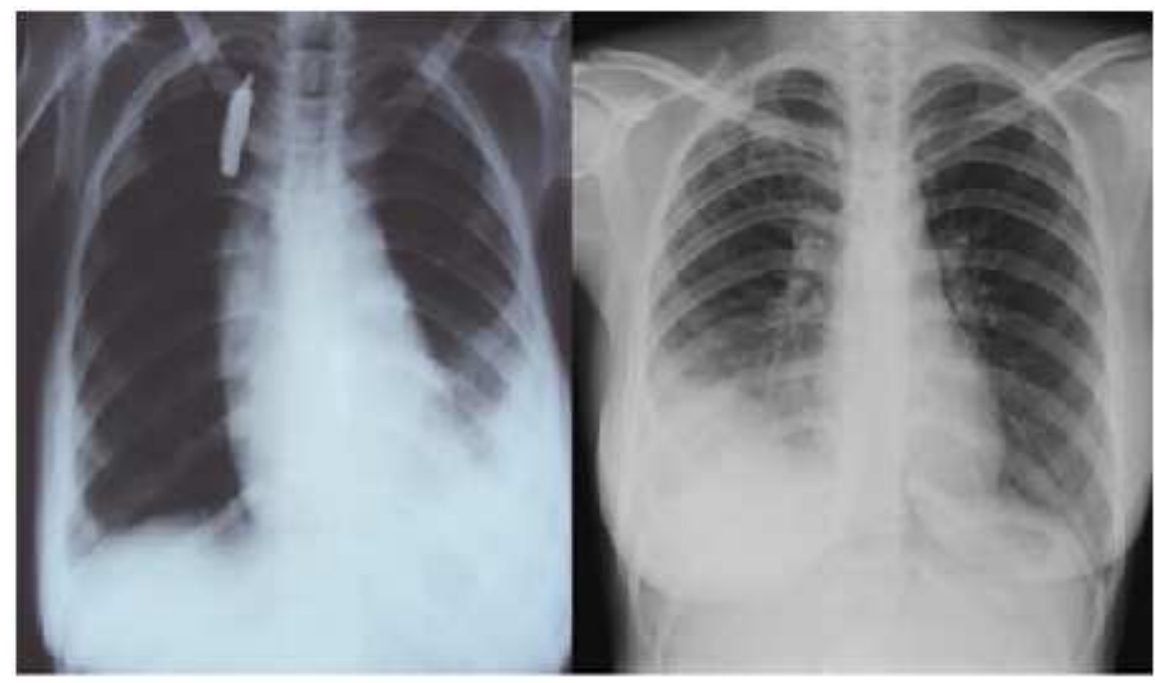

Fig.4 (a,b) X ray Chest PA View (a) Left pleural effusion with para-vertebral shadowing in patient with Pott's spine(b) Right pleural effusion,cytology proved Tuberculosis. 


\section{ORIGINAL ARTICLE}

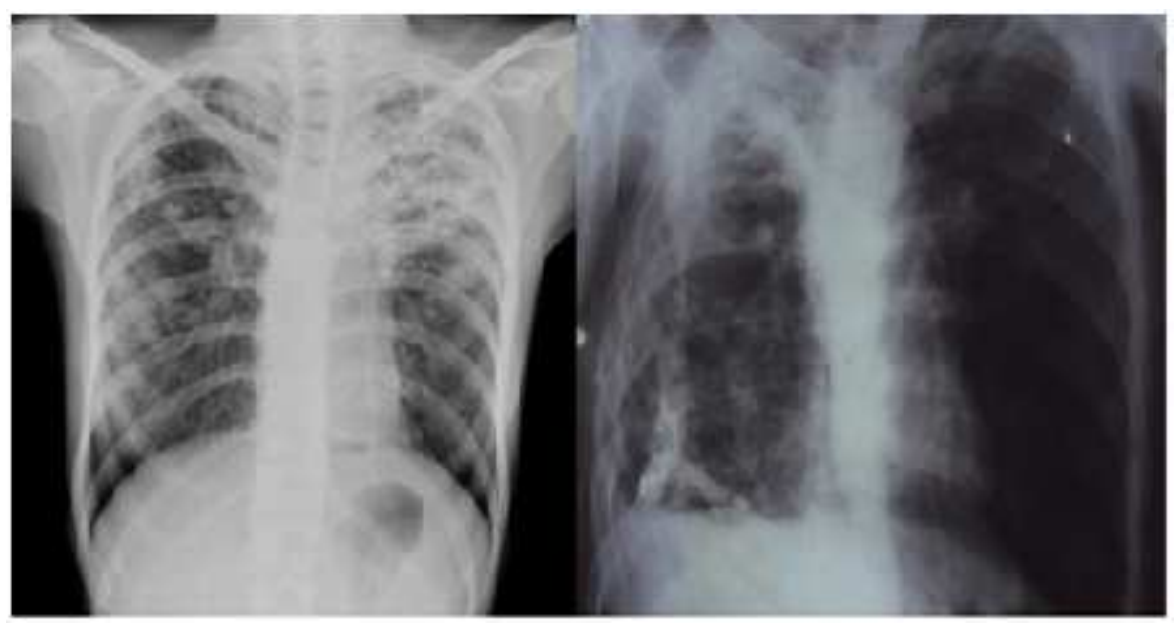

Fig. 5 (a,b)X ray Chest PA View in sputum positive patients(a) Fibro-calcification in bilateral upper zones(b) Right pleural calcification with fibrosis right lung.

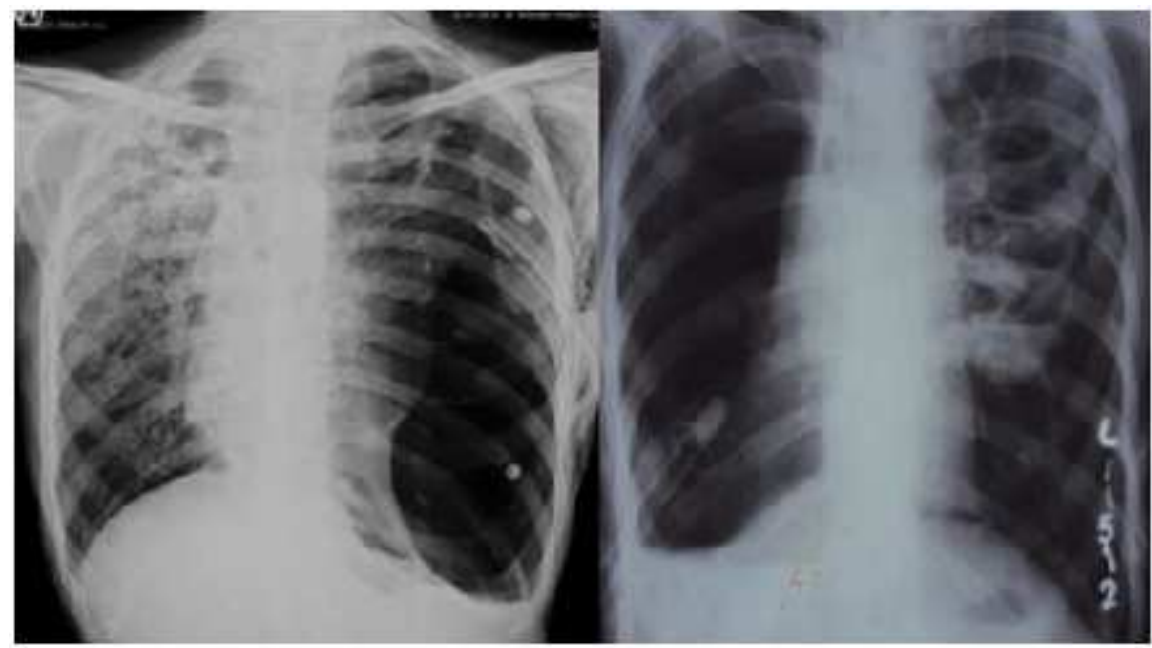

Fig. 6 (a,b) Xray Chest PA view in defaulter Pulmonary tuberculosis patients(a)Extensive left pneumothorax with eversion of diaphragm, mediastinal shift towards opposite side with fibrotic bands right apical region.

(b) Right hydro-pneumothorax with thin walled cavitatory lesion left upper and mid zones. 


\section{ORIGINAL ARTICLE}

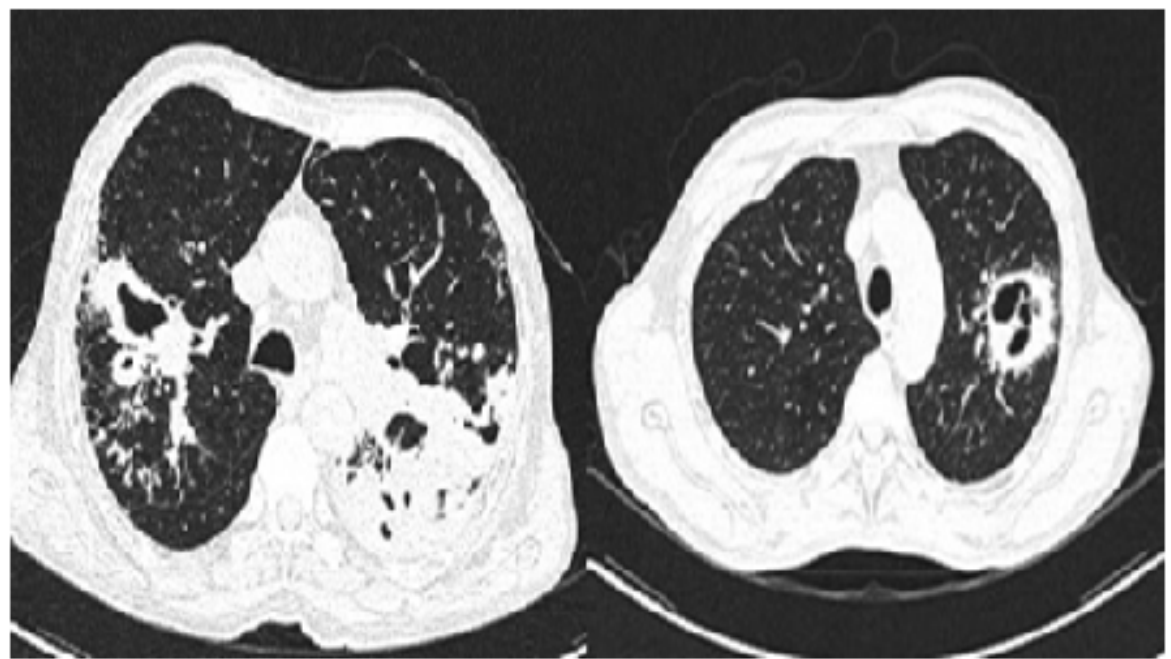

Fig. 7(a, b) MDCT Chest Axial Images in sputum positive Pulmonary tuberculosis patients. Thick, irregular walled cavitatory lesions with 'Centri-nodular' and 'tree-in-bud opacities' in surrounding parenchyma. Note the pleural extension of the disease in figure 7(a).

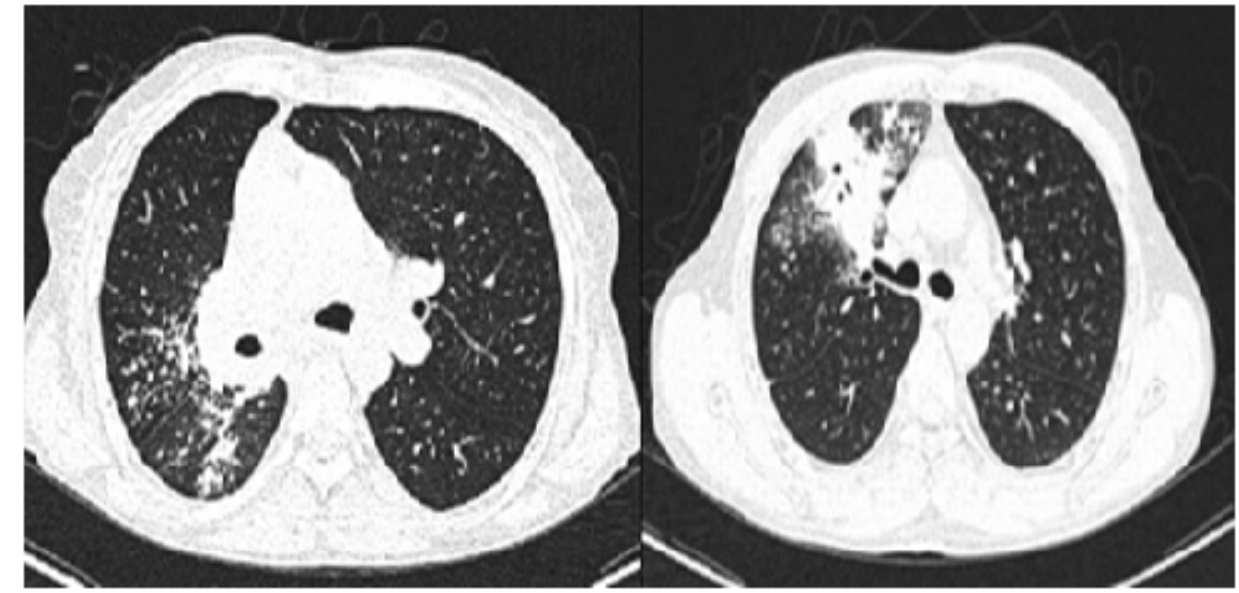

Fig. 8(a,b) MDCT Chest Axial Images in sputum positive PTB patients (a)Centrilobular nodules and 'tree-in-bud' opacities at the level of right main bronchus(b)Irregular mass like consolidation with nodular opacities in surrounding lung. 


\section{ORIGINAL ARTICLE}

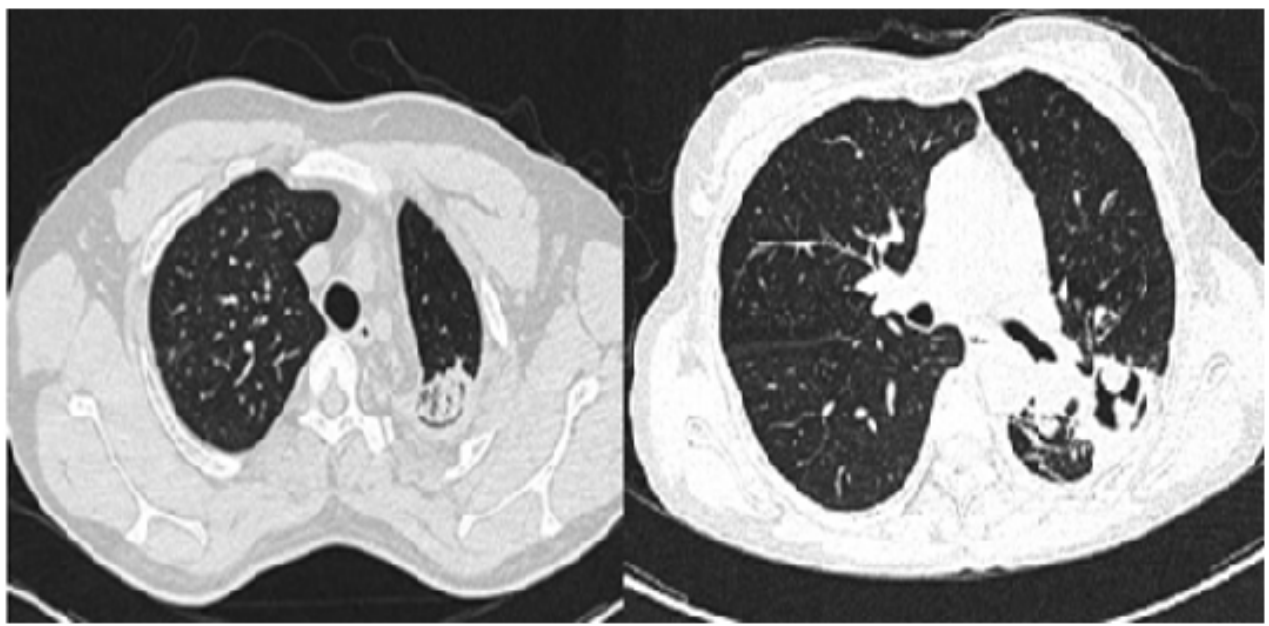

Fig. 9 (a,b) MDCT Chest-Axial Images in sputum positive Pulmonary tuberculosis patients. (a)Mass with internal sponge like architecture and air meniscus in the left upper lobe- Old T.B. cavity with fungus ball.(b) Cavity having mycelial fronds at the level of left main bronchus and centrilobular nodules in surrounding lung parenchyma.

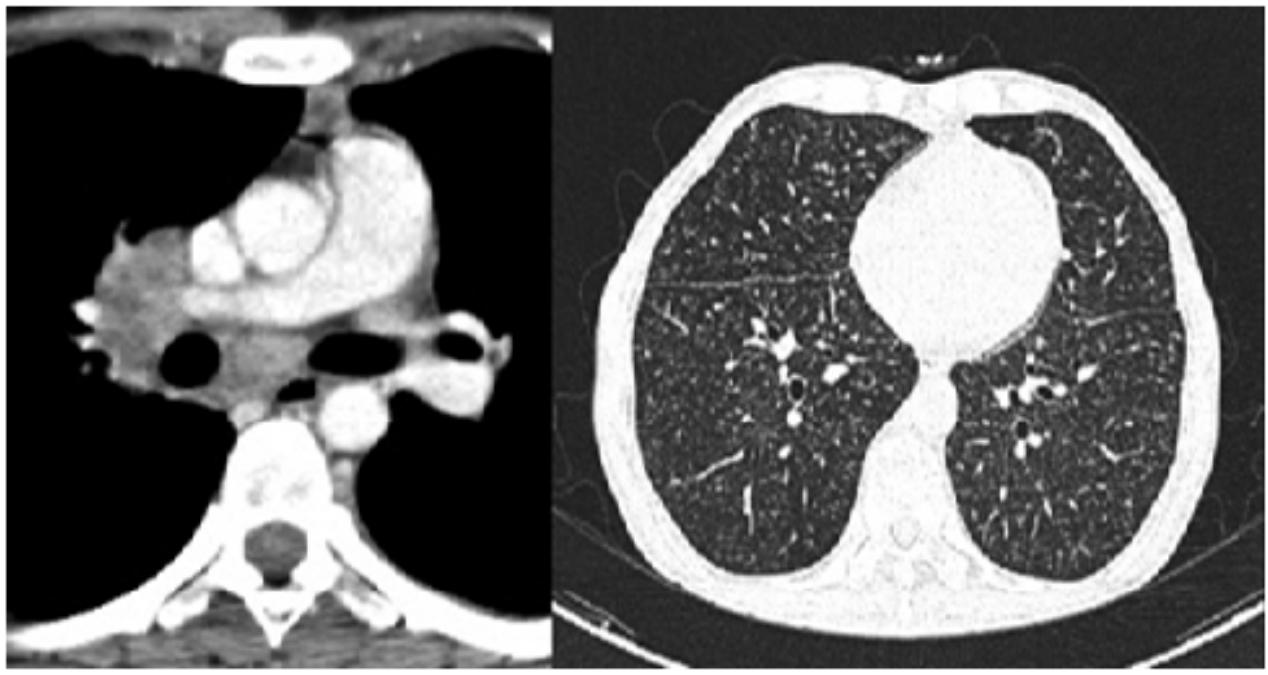

Figure 10 (a) CECT scan at the level of Pulmonary trunk (mediastinal window) showing multiple subcarinal and right hilar lymph nodes having central low attenuation area and enhancing periphery in a patient with primary Koch's.

(b)HRCT axial section at level of ventricles in a 60 years old male patient with culture positive for acid fast bacilli. Multiple, uniform-sized small nodules can be seen randomly distributed throughout the lung. Note subpleural and sub-fissural nodules -Miliary Tuberculosis. 